\title{
Precision Spectroscopy of Pionic 1s States of Sn Nuclei and Evidence for Partial Restoration of Chiral Symmetry in the Nuclear Medium
}

\author{
K. Suzuki, ${ }^{1}$ M. Fujita, ${ }^{2}$ H. Geissel, ${ }^{3}$ H. Gilg, ${ }^{4}$ A. Gillitzer, ${ }^{5}$ R. S. Hayano, ${ }^{1}$ S. Hirenzaki, ${ }^{2}$ K. Itahashi, ${ }^{6}$ M. Iwasaki, ${ }^{6}$ \\ P. Kienle, ${ }^{4,7}$ M. Matos, ${ }^{3}$ G. Münzenberg, ${ }^{3}$ T. Ohtsubo, ${ }^{8}$ M. Sato, ${ }^{9}$ M. Shindo, ${ }^{1}$ T. Suzuki, ${ }^{1}$ H. Weick, ${ }^{3}$ M. Winkler, ${ }^{3}$ \\ T. Yamazaki, ${ }^{10}$ and T. Yoneyama ${ }^{9}$ \\ ${ }^{1}$ Department of Physics, School of Science, University of Tokyo, 7-3-1 Hongo, Bunkyo-ku, Tokyo 113-0033, Japan \\ ${ }^{2}$ Department of Physics, Nara Women's University, Kita-Uoya Nishimachi, Nara 630-8506, Japan \\ ${ }^{3}$ Gesellschaft für Schwerionenforschung, D-64291 Darmstadt, Germany \\ ${ }^{4}$ Physik-Department, Technische Universität München, D-85748 Garching, Germany \\ ${ }^{5}$ Institut für Kernphysik, Forschungszentrum Jülich, D-52425 Jülich, Germany \\ ${ }^{6}$ Muon Science Laboratory, RIKEN, 2-1 Hirosawa, Wako-shi, Saitama, 351-0198 Japan \\ ${ }^{7}$ Institute for Medium Energy Physics of the Austrian Academy of Sciences, Boltzmanngasse 3, A-1090 Vienna, Austria \\ ${ }^{8}$ Department of Physics, Niigata University, 2-8050, Igarashi, Niigata-shi, Niigata, 950-2181, Japan \\ ${ }^{9}$ Department of Physics, Tokyo Institute of Technology, 2-12-1 Ookayama, Meguro-ku, Tokyo 152-8551, Japan \\ ${ }^{10}$ RI Beam Science Laboratory, RIKEN, 2-1 Hirosawa, Wako-shi, Saitama, 351-0198 Japan \\ (Received 18 November 2002; revised manuscript received 10 June 2003; published 19 February 2004)
}

Deeply bound $1 s$ states of $\pi^{-}$in ${ }^{115,119,123} \mathrm{Sn}$ were preferentially observed using the $\operatorname{Sn}\left(d,{ }^{3} \mathrm{He}\right)$ piontransfer reaction under the recoil-free condition. The $1 s$ binding energies and widths were precisely determined and were used to deduce the isovector parameter of the $s$-wave pion-nucleus potential to be $b_{1}=-(0.115 \pm 0.007) m_{\pi}^{-1}$. The observed enhancement of $\left|b_{1}\right|$ over the free $\pi N$ value $\left(b_{1}^{\text {free }} / b_{1}=\right.$ $0.78 \pm 0.05)$ indicates a reduction of the chiral order parameter, $f_{\pi}^{*}(\rho)^{2} / f_{\pi}^{2} \approx 0.64$, at the normal nuclear density, $\rho=\rho_{0}$.

DOI: $10.1103 /$ PhysRevLett.92.072302

Our work deals with characteristic properties of strongly interacting bound sytems, hadrons, consisting of light quarks $(u$ and $d$ ). Their masses are nearly 2 orders of magnitude smaller $\left(m_{u} \approx 5 \mathrm{MeV}, m_{d} \approx 8 \mathrm{MeV}\right)$ than typical hadron masses of $\approx 1 \mathrm{GeV}$. This extraordinary phenomenon is proposed to be produced by spontaneous breaking of chiral symmetry for massless quarks subject to the strong interaction [1-3]. It results in a ground state, the vacuum state, with a finite expectation value of quarkantiquark pairs, $\langle\bar{q} q\rangle_{0} \approx-(250 \mathrm{MeV})^{3}$ [4]. In such a scenario the hadrons are considered as quasiparticle excitations of the condensate, $\langle\bar{q} q\rangle$, separated by an energy gap of $\approx 1 \mathrm{GeV}$. The lowest energy excitation modes of the condensate, so-called Nambu-Goldstone bosons, are identified as pions. Their $s$-wave interaction with nucleons is predicted to vanish in its isoscalar part, and determined by the pion decay constant, $f_{\pi}^{2}$, in its isovector part $[5,6]$ which is consistent with recent experimental values $[7,8]$. The $f_{\pi}^{2}$ is also the order parameter of chiral symmetry breaking and directly connected to the magnitude of $\langle\bar{q} q\rangle$ through the Gell-Mann-Oaks-Renner relation [4].

We examine this chiral symmetry scenario by implanting a $\pi^{-}$in a nuclear medium of density $\rho[9,10]$, where a new vacuum state with a reduced condensate, $\langle\bar{q} q\rangle_{\rho}$, is proposed to be created [2]. This is the most basic case of a large effort to study the density and temperature dependence of the $\langle\bar{q} q\rangle$ in relativistic heavy-ion collisions.

The density dependence of the quark condensate is expressed in the leading order by the pion-nucleon $\sigma$
PACS numbers: 13.75.Gx, 14.40.Aq, 25.45.Hi, 36.10.Gv

term $\left(\sigma_{N} \approx 45 \mathrm{MeV}\right)$ in the following form [11]:

$$
\frac{\langle\bar{q} q\rangle_{\rho}}{\langle\bar{q} q\rangle_{0}} \approx 1-\frac{\sigma_{N}}{m_{\pi}^{2} f_{\pi}^{2}} \rho,
$$

which yields a reduction of about 0.65 for the normal nuclear density, $\rho=\rho_{0}=0.17 \mathrm{fm}^{-3}$. Likewise, the pion decay constant in a medium (identified as the time component of the axial current) is reduced as [12] $f_{\pi}^{*}(\rho)^{2} / f_{\pi}^{2} \approx 1-\alpha \rho$, where the parameter $\alpha$ is predicted to be $\alpha \rho_{0} \approx 0.45$ from the chiral dynamics [13]. This reduction of the ratio is associated with the free and the in-medium isovector $\pi N$ scattering length ( $b_{1}^{\text {free }}$ and $b_{1}^{*}$, respectively) as [14]

$$
R(\rho)=\frac{b_{1}^{\text {free }}}{b_{1}^{*}(\rho)} \approx \frac{f_{\pi}^{*}(\rho)^{2}}{f_{\pi}^{2}} \approx 1-\alpha \rho .
$$

Our program aims at measuring the isovector $\pi N$ interaction parameter in the pion-nucleus potential [ $\left.b_{1}^{*}(\rho)\right]$ [15] by studying deeply bound $1 s$ states of $\pi^{-}$ in heavy $N>Z$ nuclei [16-20]. Such states were predicted to be produced as discrete states by nuclear reactions [21-25] and to provide unique information on the $s$-wave interaction, whereas in most pionic atoms the repulsive $s$-wave interaction is nearly counterbalanced by the attractive $p$-wave interaction (see, for instance, Batty et al. [26]). In recent papers we showed that the density-dependent parameter $b_{1}^{*}(\rho)$ can be well represented by a constant parameter $b_{1}$ [27], and developed a method to deduce the $b_{1}$ parameter [28]. The data of the 
$1 s \pi^{-}$state in ${ }^{205} \mathrm{~Pb}$ [20], combined with information on $1 s$ states of light pionic atoms, yielded $b_{1}=$ $-0.116_{-0.017}^{+0.015} m_{\pi}^{-1}$. A similar conclusion was also obtained from global fits of pionic atom data by Friedman, $b_{1}=$ $-(0.122 \pm 0.004) m_{\pi}^{-1} \quad[29]$ and $-(0.113 \pm 0.004) m_{\pi}^{-1}$ [30], although some ambiguity arising from the $p$-wave part remains. In order to determine $b_{1}$ more reliably and accurately, it is essential to perform high-precision spectroscopy on deeply bound $1 s \pi^{-}$states in heavy nuclei.

In this Letter we report on systematic experimental studies of $1 s \pi^{-}$states in a series of $\mathrm{Sn}$ isotopes, which were produced with the $\operatorname{Sn}\left(d,{ }^{3} \mathrm{He}\right)$ reactions. One of the advantages of using $\mathrm{Sn}$ isotopes is that we can produce the $1 s \pi^{-}$states as the most dominant quasisubstitutional states, $(1 s)_{\pi^{-}}(3 s)_{n}^{-1}$, because of the presence of the $3 s$ orbital near the Fermi surface, as theoretically predicted [25]. Another merit is to make use of isotopes over a wide range of $(N-Z) / A$ to test the isospin dependence [10].

We used a deuteron beam from the heavy-ion synchrotron SIS at GSI, Darmstadt, combined with the fragment separator as a high-resolution forward spectrometer [18]. The present experiment had many new features and improvements. We chose the exact recoil-less condition to suppress minor states other than the enhanced $1 s \pi^{-}$ states with quasisubstitutional $1 s \pi^{-}$states, with a deuteron beam of a small momentum spread and an accurately measured energy of $503.388 \pm 0.100 \mathrm{MeV}$. Its intensity was $1.5 \times 10^{11}$ deuterons per spill. The beam was focused on a narrow spot with a width of about $1 \mathrm{~mm}$, hitting a strip target with a width of $1.5 \mathrm{~mm}$ and a thickness of $20 \mathrm{mg} / \mathrm{cm}^{2}$. Enriched Sn isotopes with $A=116$ (98.0\%), $120(99.2 \%)$, and $124(96.6 \%)$ were used as targets.

The strategy to arrive at high accuracy in determining the binding energies and widths of the $1 s$ states was the following. First, we used the nonpionic ${ }^{A} \operatorname{Sn}\left(d,{ }^{3} \mathrm{He}\right){ }^{A-1} \operatorname{In}($ g.s. $)(A=112,116,120,124)$ reactions to calibrate the spectrometer momentum (with respect to the incident beam momentum) and dispersion to achieve an accuracy of $6 \mathrm{keV}$. The overall energy resolution, which was crucial for measuring the widths of the $1 \mathrm{~s} \pi^{-}$states, was also determined from these spectra to be $394 \pm$ $33 \mathrm{keV}$ (FWHM). Second, we put a thin Mylar layer on the downstream surface of each Sn target to measure the ${ }^{3} \mathrm{He}$ peak from the $p\left(d,{ }^{3} \mathrm{He}\right) \pi^{0}$ reaction as a built-in indicator of absolute calibration. We observed spectra, $d^{2} \sigma / d E / d \Omega$, on Mylar-covered ${ }^{116} \mathrm{Sn},{ }^{120} \mathrm{Sn},{ }^{124} \mathrm{Sn}$ targets as a function of the ${ }^{3} \mathrm{He}$ kinetic energy, as shown in Fig. 1.

The skewed peaks at around $371 \mathrm{MeV}$ arise from $p\left(d,{ }^{3} \mathrm{He}\right) \pi^{0}$. Their kinematically broadened shapes were well explained by a simulation, which took into account the beam-energy profile at the thin Mylar layer, the angular distribution, the spectrometer acceptance $\left(\Delta \theta_{x} \approx\right.$ $\pm 15 \mathrm{mr}$ and $\Delta \theta_{y} \approx \pm 10 \mathrm{mr}$ ), and the kinematical energy shifts of the ${ }^{3} \mathrm{He}$ particles. It turned out that the energy at half maximum of the higher-energy edge of the peak

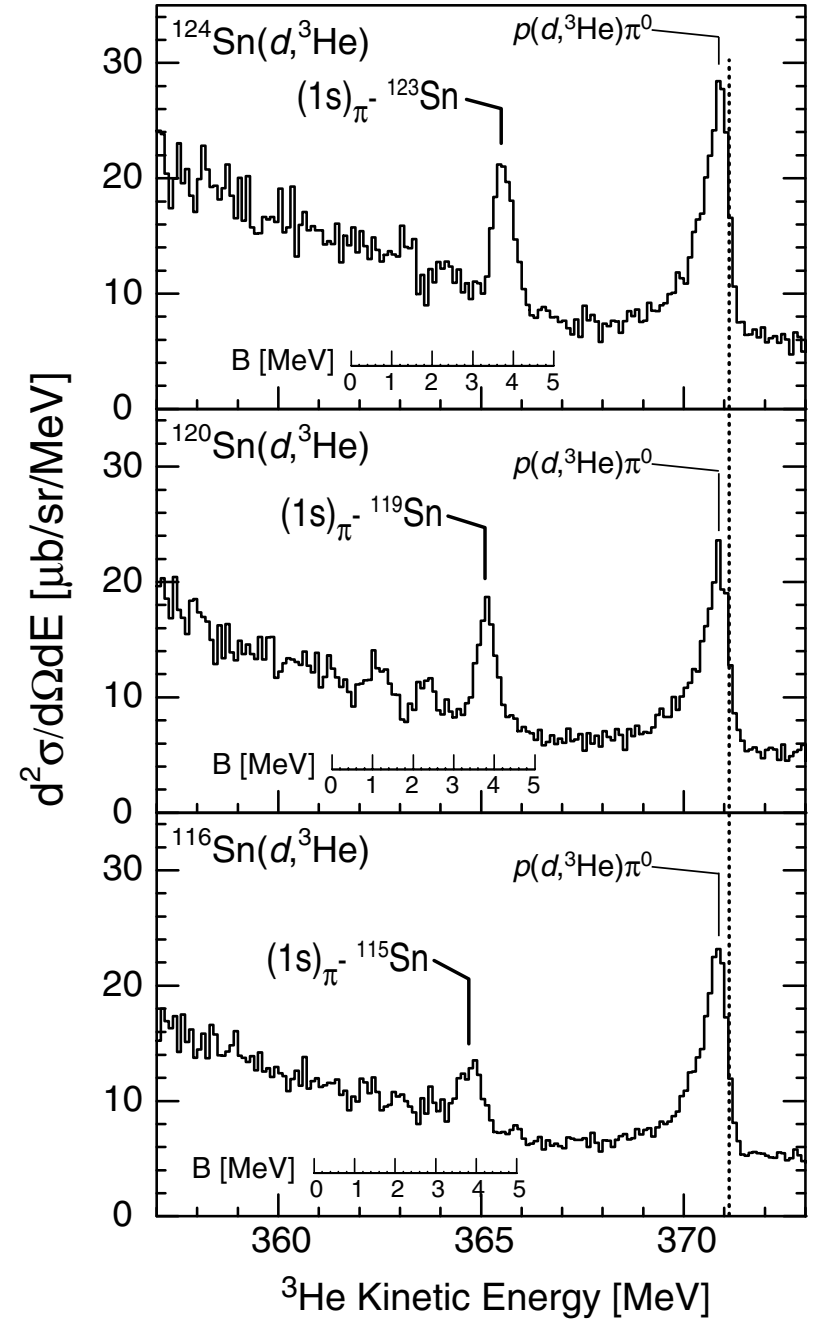

FIG. 1. Double differential cross sections versus the ${ }^{3} \mathrm{He}$ kinetic energy of the ${ }^{124,120,116} \mathrm{Sn}\left(d,{ }^{3} \mathrm{He}\right)$ reactions measured at the incident deuteron energy of $503.388 \mathrm{MeV}$. The scales of the $\pi^{-}$binding energies in ${ }^{123,119,115} \mathrm{Sn}$ are also indicated.

(shown as vertical dotted lines in Fig. 1) represents the kinetic energy of ${ }^{3} \mathrm{He}$ in the ideal case of $0^{\circ}$ emission from the $p\left(d,{ }^{3} \mathrm{He}\right) \pi^{0}$ reaction, and serves as an absolute calibration point with an accuracy of $\pm 7 \mathrm{keV}$. We adopted this "edge" calibration for our final results, since this long term calibration was pertinent to all runs of the three targets. It was found to agree within $13 \mathrm{keV}$ with the short-term calibration using the $\operatorname{Sn}\left(d,{ }^{3} \mathrm{He}\right) \operatorname{In}($ g.s. $)$ reactions.

In each spectrum of Fig. 1 one distinct peak at around $365 \mathrm{MeV}$ was observed, which was assigned to a dominant configuration of $(1 s)_{\pi}(3 s)_{n}^{-1}$. The overall spectrum shapes for the three Sn targets were found to be in good agreement with the predicted ones [25]. The spectra were decomposed according to the theoretical prescription of Ref. [25], from which we could precisely determine the $1 s$ binding energies $\left(B_{1 s}\right)$ and widths $\left(\Gamma_{1 s}\right)$. The results are summarized in Table I and in Fig. 2. The quoted errors are statistical, systematic (arising from the calibration), and 
TABLE I. Observed binding energies $\left(B_{1 s}\right)$ and widths $\left(\Gamma_{1 s}\right)$ of the $1 s \pi^{-}$states in ${ }^{115,119,123} \mathrm{Sn}$ isotopes.

\begin{tabular}{lcccccccc}
\hline \hline $\begin{array}{c}B_{1 s} \\
\text { Isotope }\end{array}$ & \multicolumn{3}{c}{$\Delta B_{1 s}(\mathrm{MeV})$} & & \multicolumn{3}{c}{$\Delta \Gamma_{1 s}(\mathrm{MeV})$} \\
$(\mathrm{MeV})$ & Stat. & Syst. & Total & $(\mathrm{MeV})$ & Stat. & Syst. & Total \\
\hline${ }^{115} \mathrm{Sn}$ & 3.906 & \pm 0.021 & \pm 0.012 & \pm 0.024 & 0.441 & \pm 0.068 & \pm 0.054 & \pm 0.087 \\
${ }^{119} \mathrm{Sn}$ & 3.820 & \pm 0.013 & \pm 0.012 & \pm 0.018 & 0.326 & \pm 0.047 & \pm 0.065 & \pm 0.080 \\
${ }^{123} \mathrm{Sn}$ & 3.744 & \pm 0.013 & \pm 0.012 & \pm 0.018 & 0.341 & \pm 0.036 & \pm 0.063 & \pm 0.072 \\
\hline \hline
\end{tabular}

combined errors. In this study isotope shifts of deeply bound pionic $1 s$ states in heavy nuclei are seen for the first time.

The most serious problem in our analysis is the relatively poor knowledge concerning the neutron distribution $\rho_{n}(r)$ in $\mathrm{Sn}$ isotopes, whereas the proton distribution $\rho_{p}(r)$ is well known [31]. We take the following form for the difference between the neutron and proton rms radii, which was derived based on experimental data of antiprotonic atoms of $\mathrm{Sn}$ isotopes [32] as well as of many other nuclei $\Delta r_{n p}=(1.01 \pm 0.15)(N-Z) / A+$ $(-0.04 \pm 0.03) \mathrm{fm}$. In converting $\Delta r_{n p}$ into the diffuseness parameters $\left(a_{p}, a_{n}\right)$ and the half-density radii $\left(c_{p}, c_{n}\right)$ in the two-parameter Fermi distribution, we took halfway between the "halo" type $\left(c_{p}=c_{n}, a_{p}<\right.$ $\left.a_{n}\right)$ and the "skin" type $\left(c_{p}<c_{n}, a_{p}=a_{n}\right)$ [32], in accordance with a proton elastic scattering result in $\mathrm{Pb}$ isotopes (see Ref. [28]). The adopted parameters $\left\{c_{p}, a_{p}, c_{n}, a_{n}\right\}$ in $\mathrm{fm}$ are $\{5.405,0.523,5.473,0.552\}$ for ${ }^{115} \mathrm{Sn},\{5.442,0.523,5.532,0.560\}$ for ${ }^{119} \mathrm{Sn}$, and $\{5.484,0.523,5.594,0.569\}$ for ${ }^{123} \mathrm{Sn}$.

The influence of $\Delta r_{n p}$ on the extraction of optical potential parameters in global fits of pionic atom data

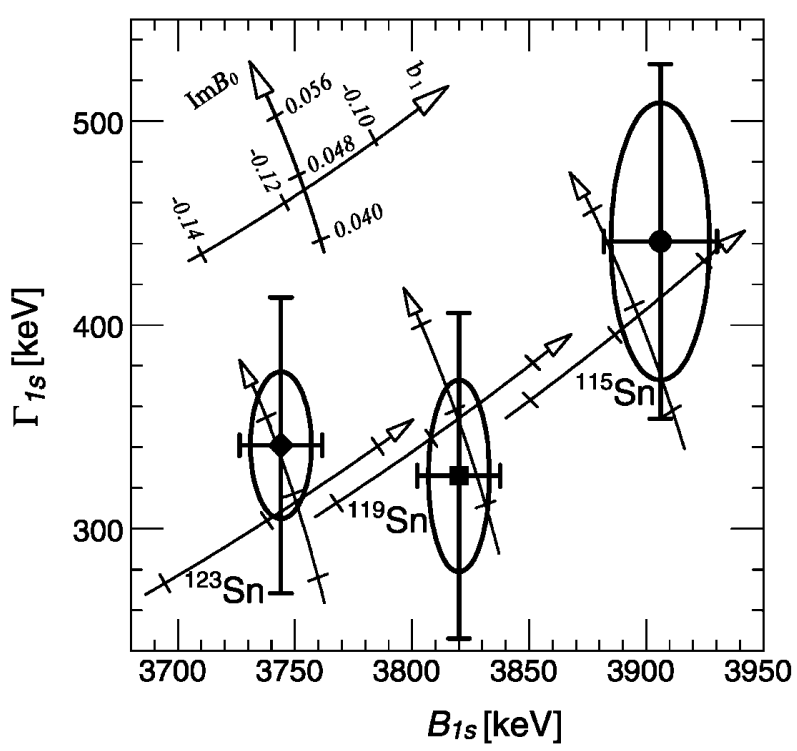

FIG. 2. Experimental values of $\left\{B_{1 s}, \Gamma_{1 s}\right\}$ (elliptic circles for statistical errors, horizontal and vertical bars for total errors) for $\pi^{-}$in ${ }^{115,119,123} \mathrm{Sn}$, shown together with theoretical relations with $\left\{b_{1}, \operatorname{Im} B_{0}\right\}$ for each isotope. was recently discussed in detail by Friedman and Gal [33] based on old theoretical values of $\Delta r_{n p}$ [34], which are considerably different from the empirical ones [32] we use. These values are supported by a new protonscattering result on $\mathrm{Sn}$ isotopes [35] and also by new relativistic Hartree-Fock-Bogoliubov calculations [36]. In our view the use, in our analysis, of the experimental data available now resolves the concerns of Ref. [33] with regard to our results.

We made simultaneous fitting of $B_{1 s}$ and $\Gamma_{1 s}$ of the three $\mathrm{Sn}$ isotopes together with those of symmetric light nuclei, leaving $b_{0}, b_{1}, \operatorname{Re} B_{0}$, and $\operatorname{Im} B_{0}$ as free parameters. Since the $1 s$ binding energy is insensitive to the $p$-wave potential, we could safely fix the $p$-wave parameters to the known values from pionic atom data [26]. Hereafter, the units of $m_{\pi}^{-1}$ for $b_{0}, b_{1}$ and of $m_{\pi}^{-4}$ for $B_{0}$ will be omitted. We chose ${ }^{16} \mathrm{O},{ }^{20} \mathrm{Ne}$, and ${ }^{28} \mathrm{Si}$ for which the nuclear parameters are well known and the condition $\rho_{p}(r)=\rho_{n}(r)$ is clearly fulfilled. Thus, the obtained values are $b_{0}=-0.0233 \pm 0.0038, \quad b_{1}=-0.1149 \pm$ $0.0074, \operatorname{Re} B_{0}=-0.019 \pm 0.017$, and $\operatorname{Im} B_{0}=0.0472 \pm$ 0.0013 . The errors include both statistical and systematic uncertainties. In Fig. 2 we show the obtained relations of $\left\{b_{1}, \operatorname{Im} B_{0}\right\}$ mapped on $\left\{B_{1 s}, \Gamma_{1 s}\right\}$ for each $\mathrm{Sn}$ isotope. The intersections of the tilted axes are chosen to the best-fit values of $\left\{b_{1}, \operatorname{Im} B_{0}\right\}$. We also show likelihood contours in the plane of $\left\{b_{1}, \operatorname{Im} B_{0}\right\}$ in Fig. 3 .

The smallness of $\operatorname{Re} B_{0}(-0.019 \pm 0.017)$ supports the value $(-0.038 \pm 0.025)$ claimed from the requirement that the effective $b_{0}$ consists of the free $\pi N$ value and the double-scattering term [28]. The combined isoscalar parameter, $b_{0}+0.215 \operatorname{Re} B_{0}=-0.0274 \pm 0.0002$, is in good agreement with that obtained in the analysis of the ${ }^{205} \mathrm{~Pb}$ [28]. The imaginary part, $\operatorname{Im} B_{0}=0.0472 \pm$ 0.0013 , is consistent with the global-fit value of $0.055 \pm$ 0.003 by Batty et al. [26] and Friedman [29,30], considering that they included the angle-transformation (AT) term, which causes an appreciable decrease in the width [27]. In fact, the best-fit value in our analysis with the AT term included is $\operatorname{Im} B_{0}=0.058 \pm 0.003$. These isoscalar parameters are in fine agreement with those determined by using only the $1 s$ states of light symmetric pionic atoms. The addition of the Sn data consequently yields a precise value for $b_{1}$.

Figure 3 also shows how the best-fit values move, if we choose the halo type (open square) or the skin type (open diamond) for $\rho_{n}(r)-\rho_{p}(r)$. Furthermore, we find that the possible change of $b_{1}$, when we allowed an 


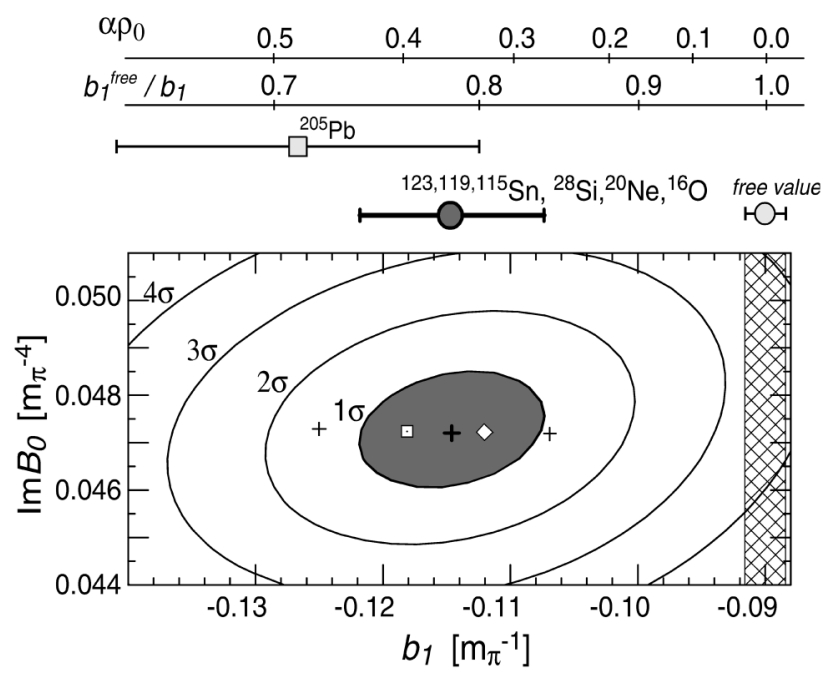

FIG. 3. Likelihood contours in the $\left\{b_{1}, \operatorname{Im} B_{0}\right\}$ plane from the simultaneous fitting of $\left\{B_{1 s}, \Gamma_{1 s}\right\}$ of the $1 s$ pionic states in the three $\mathrm{Sn}$ isotopes and three light symmetric nuclei. The previous ${ }^{205} \mathrm{~Pb}$ data reanalyzed with $\operatorname{Im} B_{0}(=0.046)$ is shown for comparison.

uncertainty of $\pm 0.04 \mathrm{fm}$ in $\Delta r_{n p}$ [32], would be around \pm 0.009 , as indicated by two crosses in the figure.

The magnitude of the observed $\left|b_{1}\right|$ is significantly enhanced over the free $\pi N$ value, which translates into a reduction of $f_{\pi}^{* 2}$ [Eq. (2)] as

$$
\begin{gathered}
R=b_{1}^{\text {free }} / b_{1}=0.78 \pm 0.05 \\
\approx b_{1}^{\text {free }} / b_{1}^{*}\left(\rho_{e}\right) \approx f_{\pi}^{*}\left(\rho_{e}\right)^{2} / f_{\pi}^{2} \approx 1-\alpha \rho_{e},
\end{gathered}
$$

where we used the fact $[27,28]$ that the solution with a local-density-dependent parameter, $b_{1}^{*}(\rho)=b_{1}^{\text {free }} /[1-$ $\alpha \rho(r)]$, is equivalent to that using a corresponding constant parameter $b_{1}=b_{1}^{\text {free }} /\left(1-\alpha \rho_{e}\right)$ with an effective density $\rho_{e} \approx 0.6 \rho_{0}$.

The above value hence implies that the chiral order parameter, $f_{\pi}^{*}(\rho)^{2}$, would be reduced by a factor of $\approx 0.64$ at the normal nuclear density $\rho=\rho_{0}$. Using Eq. (2) in the analysis, we obtain an experimental value of $\alpha \rho_{0} \approx 0.36$, as shown in Fig. 3, which is close to the value 0.45 predicted by chiral perturbation theory [13]. If a theoretical value, $m_{\pi}^{*} \approx m_{\pi}+3 \mathrm{MeV}$ (averaged over $\pi^{+}$and $\pi^{-}$ [13]), is inserted into an in-medium Gell-Mann-OakesRenner relation [2,12], $\langle\bar{q} q\rangle_{\rho_{0}} /\langle\bar{q} q\rangle_{0}$ will be $\left(m_{\pi}^{*} / m_{\pi}\right)^{2} \times$ $\left(1-\alpha \rho_{0}\right) \approx 0.67$, which is in good agreement with the value of 0.65 , as predicted by using Eq. (1). We have thus found clear evidence for the partial restoration of chiral symmetry, probed by well-defined pionic states in a welldefined nuclear density.

The authors thank the staff of GSI for the continuous efforts to provide superb experimental conditions and Professor H. Toki, Professor W. Weise, and Professor T. Hatsuda for stimulating and encouraging discussions. This work is supported by Grants-in-Aid for Scientific
Research of Monbukagakusho (Japan) and Japan Society for the Promotion of Science, and by the Bundesministerium für Bildung, Wissenschaft, Forschung und Technologie (Germany) and the Gesellschaft für Schwerionenforschung Darmstadt.

[1] Y. Nambu and G. Jona-Lasinio, Phys. Rev. 122, 345 (1961); 124, 246 (1961).

[2] See T. Hatsuda and T. Kunihiro, Phys. Rep. 247, 221 (1994), and references therein.

[3] U. Vogl and W. Weise, Prog. Part. Nucl. Phys. 27, 195 (1991).

[4] M. Gell-Mann, R. J. Oakes, and B. Renner, Phys. Rev. 175, 2195 (1968).

[5] Y. Tomozawa, Nuovo Cimento A 46, 707 (1966).

[6] S. Weinberg, Phys. Rev. Lett. 17, 616 (1966).

[7] H.-Ch. Schröder et al., Phys. Lett. B 469, 25 (1999).

[8] T. E. O. Ericson et al., Nucl. Phys. A684, 380 (2001).

[9] W. Weise, Acta Phys. Pol. B 31, 2715 (2000); Nucl. Phys. A690, 98 (2001).

[10] P. Kienle and T. Yamazaki, Phys. Lett. B 514, 1 (2001).

[11] E. G. Drukarev and E. M. Levin, Nucl. Phys. A511, 679 (1990).

[12] Y. Thorsson and A. Wirzba, Nucl. Phys. A589, 633 (1995).

[13] See U.-G. Meissner, J. A. Oller, and A. Wirzba, Ann. Phys. (N.Y.) 297, 27 (2002), and references therein.

[14] E. E. Kolomeitsev, N. Kaiser, and W. Weise, Phys. Rev. Lett. 90, 092501 (2003).

[15] M. Ericson and T. E. O. Ericson, Ann. Phys. (N.Y.) 36, 323 (1966)

[16] T. Yamazaki et al., Z. Phys. A 355, 219 (1996).

[17] T. Yamazaki et al., Phys. Lett. B 418, 246 (1998).

[18] H. Gilg et al., Phys. Rev. C 62, 025201 (2000).

[19] K. Itahashi et al., Phys. Rev. C 62, 025202 (2000).

[20] H. Geissel et al., Phys. Rev. Lett. 88, 122301 (2002).

[21] H. Toki and T. Yamazaki, Phys. Lett. B 213, 129 (1988).

[22] H. Toki et al., Nucl. Phys. A501, 653 (1989).

[23] H. Toki, S. Hirenzaki, and T. Yamazaki, Nucl. Phys. A530, 679 (1991).

[24] S. Hirenzaki, H. Toki, and T. Yamazaki, Phys. Rev. C 44, 2472 (1991).

[25] Y. Umemoto et al., Phys. Rev. C 62, 024606 (2000).

[26] C. Batty, E. Friedman, and A. Gal, Phys. Rep. 287, 385 (1997).

[27] T. Yamazaki and S. Hirenzaki, Phys. Lett. B 557, 20 (2003).

[28] H. Geissel et al., Phys. Lett. B 549, 64 (2002).

[29] E. Friedman, Phys. Lett. B 524, 87 (2002).

[30] E. Friedman, Nucl. Phys. A710, 117 (2002).

[31] G. Fricke et al., At. Data Nucl. Data Tables 60, 177 (1995).

[32] A. Trzcińska et al., Phys. Rev. Lett. 87, 082501 (2001).

[33] E. Friedman and A. Gal, Nucl. Phys. A724, 143 (2003).

[34] G. Lalazissis et al., At. Data Nucl. Data Tables 71, 1 (1999)

[35] H. Takeda et al., AIP Conf. Proc. 675, 720 (2000).

[36] T. Nikšić et al., Phys. Rev. C 66, 024306 (2002). 